\title{
QoS-Constrained Sum-Harvested Energy Maximization in OFDMA-based Wireless Cooperative Networks
}

\author{
Sumit Gautam $^{\dagger}$, Eva Lagunas ${ }^{\dagger}$, Satyanarayana Vuppala ${ }^{\S}$, Symeon Chatzinotas ${ }^{\dagger}$ and Björn Ottersten ${ }^{\dagger}$ \\ $\dagger$ Interdisciplinary Centre for Security, Reliability and Trust (SnT), University of Luxembourg, Luxembourg. \\ ${ }^{\S}$ United Technologies Research Center, Cork City, Co. Cork, Republic of Ireland. \\ Email: ${ }^{\dagger}\left\{\right.$ sumit.gautam,eva.lagunas,symeon.chatzinotas,bjorn.ottersten\}@uni.lu, ${ }^{\S}$ vuppalsa@utrc.utc.com
}

\begin{abstract}
We investigate the performances of the time-switching (TS) and power-splitting (PS) based energy harvesting models in a two-hop relay assisted network where the end-users are capable of decoding information and harvesting energy concurrently. In particular, we consider joint resource allocation and relay selection to realize Simultaneous Wireless Transmission of Information and Energy (Wi-TIE) in a multi-carrier multi-user cooperative system where the relays employ the amplify-and-forward (AF) protocol. First, we formulate based on the TS and PS Wi-TIE architectures an optimization problem to maximize the sum of energy harvested at the end-users, taking into consideration each user's quality-of-service (QoS) requirement as well as power constraints at the transmit and relaying nodes. We then solve the formulated problem to optimize the users' Wi-TIE splitting factors along with relay-user coupling, subcarrier-user assignment, sub-carrier pairing, and power allocation. Finally, we demonstrate the benefits of the proposed framework via numerical results.
\end{abstract}

\section{INTRODUCTION}

Owing to the increasing performance and capacity demands in future, the adaptation of devices (facilitating wireless communication) to such requests has imposed high energy demands due to their complex hardware circuitry. In addition, given the limited life-time of the batteries, it becomes very difficult to perform high computational tasks for longer durations. This inturn forces the energy-efficiency management protocols to limit the system performance to sub-optimally lower levels. Energy harvesting (EH) from the same radio-frequency (RF) signal (as for data transmission), is a promising solution to meet the future energy needs [1].

Simultaneous Wireless Transmission of Information and Energy (Wi-TIE) has been recently introduced with focus on joint data decoding and energy harvesting at the receivers [2], [3]. In this context, two of the widely adopted Wi-TIE receiver architectures are time-switching (TS) and power-splitting (PS). In TS, the primary focus is on utilizing a fraction of the overall time period for energy harvesting followed by data processing in the remaining fractional duration of the time period using the whole signal power. On the other hand, PS focuses on splitting the received signal power into two streams for information decoding and energy harvesting, respectively.

Cooperative relaying has emerged as another promising technique to improve the overall coverage, and hence the throughput at the end-user [4]. In a scenario where the receiving node is placed very far apart from the transmitter, several devices within the network may come in handy as they may act as relays to assist in the communication process. Regenerative (e.g., decodeand-forward (DF) [5]), and non-regenerative (e.g., amplify-andforward (AF) [6]) are the two widely adopted relaying strategies in practice. The non-regenerative strategy has been widely adopted in literature due to its easy implementation and flexibility in selection of modulation schemes [7].

Recent technologies and standards like Wi-TIE and cooperative networking have been shown to infuse tremendous improvement in the system performance, when incorporated with one of the widely adopted transmission techniques, known as orthogonal frequency division multiple access (OFDMA) [8], [9]. When combined with Wi-TIE and cooperative systems, OFDMA does not only retains its existing benefits, but it also helps in providing extended coverage via relays to facilitate Wi-TIE better. In particular, the power transfer distance is largely limited by the power sensitivity of the energy harvester, which considering the current state-of-the-art technology is of $-10 \mathrm{dBm}$, significantly tighter than the $-60 \mathrm{dBm}$ assumed for effective information receivers [10].

Several works considering OFDMA resource allocation both in conventional and relay-aided communication systems have been carried out in the literature [11], [12]. However, these works do not consider the energy harvesting constraints. The benefits of cooperative transmission for OFDMA-Wi-TIE are much less investigated [13]. Recently, relay selection along with computation of optimal Wi-TIE splitting factors has been addressed in [14], [15] considering a two-hop relaying network with single user having the energy harvesting constraint. Moreover, these works have primarily focused on increasing the data rate whereas they do not study the maximization of total harvested energy by the users in a multi-carrier multi-user based cooperative networks.

The main contributions of this paper are four-fold, listed as follows.

1) Firstly, we consider a dual-hop scenario where single transmitter transfers both information and energy to multiple users with assistance from multiple half-duplex AF relays. The endusers are equipped with either a TS or a PS-based Wi-TIE receiver architecture. In this regard, we propose a novel relay selection and resource allocation technique which optimizes the network resources while easing the synchronization and control process among the relays.

2) Secondly, we formulate an optimization problem for relay selection, carrier assignment in the two hops, sub-carrier pairing, sub-carrier power allocation, and the Wi-TIE splitting factors for each user in order to maximize the sum of energy harvested at the users subjected to minimum QoS constraint at each user and power limitations at the transmitter and relays.

3) Thirdly, we propose an Energy Yield Escalation (EYE) algorithm with polynomial computational time-complexity and good performance, with sub-optimal results.

4) Finally, we demonstrate the effectiveness of the proposed method where significant gains are observed in comparison 
IEEE International Conference on Advanced Networks and Telecommunications Systems (IEEE ANTS'18), 16-19 Dec'18, Indore, India

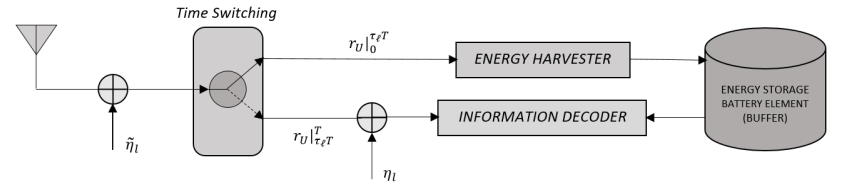

Fig. 1: TS-based Wi-TIE receiver architecture for users.

with a fixed-allocation approach. In this vein, the impacts of varying the key system parameters is observed via numerical results. Additionally, the benefits of the proposed technique are provided and some possible future directions of this work are discussed.

Further sections of this paper are organized as follows. Section II provides an introduction to the system model. The problem formulation and the proposed solution are presented in Section III and Section IV, respectively. Brief analysis on the computational complexities of proposed methods is provided in Section V. Numerical results are shown in Section VI, followed by concluding remarks in Section VII.

\section{System Model}

We consider a half-duplex wireless cooperative network with single transmitter $\mathcal{T}$, which jointly transmits data and energy intended for $L$ users $\left(\mathcal{U}_{1}, \ldots, \mathcal{U}_{L}\right)$ with the assistance from $\mathrm{K}$ relays $\left(\mathcal{R}_{1}, \ldots, \mathcal{R}_{K}\right)$ employing the AF protocol, where $K \geq L$, in general. Each device in the system is equipped with single antenna. Due to extreme attenuation suffered by the direct transmitter-users' link, the contribution from the direct link is considered negligible and is hence discarded in this paper. The end-users are capable of performing data processing as well as energy harvesting simultaneously using a TS or PS architecture as depicted in Fig. 1 and Fig. 2, respectively. In TS, for a given block transmission of duration $T$ seconds with $T=\hat{N} T_{s}(\hat{N}$ and $T_{s}$ corresponding to the number of transmitted symbols per block and the symbol period, respectively), we define a time-switching ratio at the $\ell$-th user as $\tau_{\ell}$, where $0 \leq \tau_{\ell} \leq 1$ and $\ell=1,2, \ldots, L$, such that the energy is harvested from the received signal for the first $\tau_{\ell} \hat{N} T_{s}$ second while data processing takes place for the remaining duration. On the other hand, in case of PS, a power splitter is employed so that a fraction $\sqrt{\rho_{\ell}}$, where $0 \leq \rho_{\ell} \leq 1$, of the received signal power is used for energy harvesting, while the remaining is sent to the information decoder.

The overall communication process takes place in two orthogonal time phases where the relays receive the signal from the transmitter over different OFDMA sub-carriers in the first phase while the selected relays amplify-and-forward the relevant signal to the intended users over the second phase. In order to reduce the synchronization process and complexity among the relays, each user is coupled with single relay, which is not shared with any other node. Given $N$ OFDMA sub-carriers, we denote $n \in \mathbb{Z}$ as the OFDMA sub-carrier index in the first hop such that $1 \leq n \leq N$, and $n^{\prime} \in \mathbb{Z}$ as the OFDMA sub-carrier index in the second hop, where $1 \leq n^{\prime} \leq N$. To facilitate the transmission in both the hops, an exclusive sub-carrier pairing $\left(n, n^{\prime}\right)$ is constructed at each of the chosen AF relays, where $n$ and $n^{\prime}$ may or may not be equal. Additionally, each relay is restricted to have unique sub-carrier pairs such that each subcarrier pair is assigned to one and only one relay. However, a relay may have multiple sub-carrier pairs, exclusive to itself.

The first hop channel gain coefficient between $\mathcal{T}$ and $\mathcal{R}_{k}$ on the $n$-th OFDMA sub-carrier is denoted as $g_{1, n, k}$ while the second

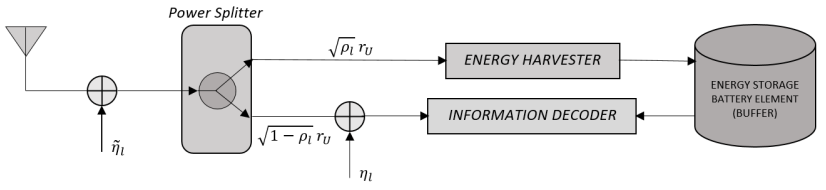

Fig. 2: PS-based Wi-TIE receiver architecture for users.

hop channel gain coefficient between the $\mathcal{R}_{k}$ and $\mathcal{U}_{\ell}$ on the $n^{\prime}$ -

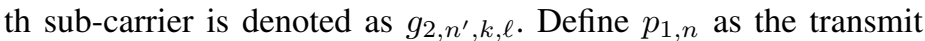
power at $\mathcal{T}$ on the $n$-th sub-carrier in the first hop. In order to re-transmit the signal, the following amplification coefficient is used at the $k$-th relay

$$
w_{\left(n, n^{\prime}\right), k}=\sqrt{\frac{p_{2, n^{\prime}, k}}{p_{1, n}\left|g_{1, n, k}\right|^{2}+\sigma_{k}^{2}}},
$$

which guarantees that $\mathcal{R}_{k}$ transmits with a power $p_{2, n^{\prime}, k}$ on the $n^{\prime}$-th sub-carrier. In (1), the noise power at $\mathcal{R}_{k}$ is denoted by $\sigma_{k}^{2}$. Let $P_{\mathcal{T}}$ and $P_{\mathcal{R}_{k}}$ be the limitation on total available power at $\mathcal{T}$ and $\mathcal{R}_{k}$, respectively.

For simplicity, we normalize the transmission time in both the hops to refer to the power and energy terms interchangeably. The antenna noise, $\tilde{\eta}_{\ell} \in \mathcal{C N}\left(0, \sigma_{\tilde{\eta}_{\ell}}^{2}\right)$, is ignored in the following analysis due to its extremely lower value in comparison to the noise generated by the baseband processing circuit [16]. The relays do not harvest any energy as they are assumed to be a part of the infrastructure having their own power supply.

Considering the $\mathcal{T} \rightarrow \mathcal{R}_{k} \rightarrow \mathcal{U}_{\ell}$ link, the effective signal-tonoise ratio (SNR) observed for the $\left(n, n^{\prime}\right)$ sub-carrier pair at the decoding branch of $\mathcal{U}_{\ell}$ corresponding to the TS and PS models is respectively given by,

$$
\begin{array}{r}
\Upsilon_{\left(n, n^{\prime}\right), k, \ell}^{T S}=\frac{p_{1, n}\left|g_{1, n, k} w_{\left(n, n^{\prime}\right), k} g_{2, n^{\prime}, k, \ell}\right|^{2}}{\sigma_{k}^{2}\left|g_{2, n^{\prime}, k, \ell} w_{\left(n, n^{\prime}\right), k}\right|^{2}+\sigma_{\eta_{\ell}}^{2}}, \\
\Upsilon_{\left(n, n^{\prime}\right), k, \ell}^{P S}=\frac{\left(1-\rho_{\ell}\right) p_{1, n}\left|g_{1, n, k} w_{\left(n, n^{\prime}\right), k} g_{2, n^{\prime}, k, \ell}\right|^{2}}{\left(1-\rho_{\ell}\right) \sigma_{k}^{2}\left|g_{2, n^{\prime}, k, \ell} w_{\left(n, n^{\prime}\right), k}\right|^{2}+\sigma_{\eta_{\ell}}^{2}},
\end{array}
$$

where the down-conversion procedure at $\mathcal{U}_{\ell}$ introduces a noise power of $\sigma_{\eta_{\ell}}^{2}$. Further, we simplify the above expressions and re-write them as follows

$$
\begin{array}{r}
\Upsilon_{\left(n, n^{\prime}\right), k, \ell}^{T S}=\frac{\Upsilon_{1, n, k} \Upsilon_{2, n^{\prime}, k, \ell}}{1+\Upsilon_{1, n, k}+\Upsilon_{2, n^{\prime}, k, \ell}}, \\
\Upsilon_{\left(n, n^{\prime}\right), k, \ell}^{P S}=\frac{\left(1-\rho_{\ell}\right) \Upsilon_{1, n, k} \Upsilon_{2, n^{\prime}, k, \ell}}{1+\Upsilon_{1, n, k}+\left(1-\rho_{\ell}\right) \Upsilon_{2, n^{\prime}, k, \ell}},
\end{array}
$$

where $\Upsilon_{1, n, k}=\frac{p_{1, n}\left|g_{1, n, k}\right|^{2}}{\sigma_{k}^{2}}$, and $\Upsilon_{2, n^{\prime}, k, \ell}=\frac{p_{2, n^{\prime}, k}\left|g_{2, n^{\prime}, k, \ell}\right|^{2}}{\sigma_{\eta_{\ell}}^{2}}$. For the considered scenario, we assume that the SNR of the relayed signal is high enough. This allows further simplification of the expressions by applying the following approximation to make the problem more tractable [17], [18],

$$
\begin{gathered}
\hat{\Upsilon}_{\left(n, n^{\prime}\right), k, \ell}^{T S} \approx \frac{\Upsilon_{1, n, k} \Upsilon_{2, n^{\prime}, k, \ell}}{\Upsilon_{1, n, k}+\Upsilon_{2, n^{\prime}, k, \ell}} \\
\hat{\Upsilon}_{\left(n, n^{\prime}\right), k, \ell}^{P S} \approx \frac{\left(1-\rho_{\ell}\right) \Upsilon_{1, n, k} \Upsilon_{2, n^{\prime}, k, \ell}}{\Upsilon_{1, n, k}+\Upsilon_{2, n^{\prime}, k, \ell}} \\
\approx\left(1-\rho_{\ell}\right) \hat{\Upsilon}_{\left(n, n^{\prime}\right), k, \ell}^{T S}
\end{gathered}
$$

Regarding the harvested energy, the energy yield over the $\mathcal{T} \rightarrow$ $\mathcal{R}_{k} \rightarrow \mathcal{U}_{\ell}$ link for $\left(n, n^{\prime}\right)$ sub-carrier pair is given by,

$$
\begin{aligned}
& E_{\left(n, n^{\prime}\right), k, \ell}^{T S}=\zeta \tau_{\ell}\left[\left|w_{\left(n, n^{\prime}\right), k} g_{2, n^{\prime}, k, \ell}\right|^{2}\left(p_{1, n}\left|g_{1, n, k}\right|^{2}+\sigma_{k}^{2}\right)\right], \\
& E_{\left(n, n^{\prime}\right), k, \ell}^{P S}=\zeta \rho_{\ell}\left[\left|w_{\left(n, n^{\prime}\right), k} g_{2, n^{\prime}, k, \ell}\right|^{2}\left(p_{1, n}\left|g_{1, n, k}\right|^{2}+\sigma_{k}^{2}\right)\right],
\end{aligned}
$$

corresponding to the TS and PS Wi-TIE architectures, where $\zeta$ is 
the energy conversion efficiency of the receiver. Using (1), these expressions can be further simplified as

$$
\begin{aligned}
& E_{\left(n, n^{\prime}\right), k, \ell}^{T S}=\zeta \cdot \tau_{\ell} \cdot p_{2, n^{\prime}, k}\left|g_{2, n^{\prime}, k, \ell}\right|^{2}, \\
& E_{\left(n, n^{\prime}\right), k, \ell}^{P S}=\zeta \cdot \rho_{\ell} \cdot p_{2, n^{\prime}, k}\left|g_{2, n^{\prime}, k, \ell}\right|^{2} .
\end{aligned}
$$

Furthermore, we define the following triplet in order to simplify the notations,

$$
\left(\hat{\Upsilon}_{\left(n, n^{\prime}\right), k, \ell} ; \hat{E}_{\left(n, n^{\prime}\right), k, \ell} ; \theta_{\ell}\right)=\left\{\begin{array}{l}
\left(\hat{\Upsilon}_{\left(n, n^{\prime}\right), k, \ell}^{T S} ; E_{\left(n, n^{\prime}\right), k, \ell}^{T S} ; \tau_{\ell}\right), \\
\left(\hat{\Upsilon}_{\left(n, n^{\prime}\right), k, \ell}^{P S} ; E_{\left(n, n^{\prime}\right), k, \ell}^{P S} ; \rho_{\ell}\right),
\end{array}\right.
$$

which corresponds to the TS and PS schemes, respectively.

\section{PRoblem Formulation}

In this section, we formulate an optimization problem to maximize the sum of overall harvested energy by the users subject to individual user's QoS constraint and transmit power limitations at the transmitter and the relays. In this vein, we intend to optimize the relay selection and resource allocation along with computation of power in each sub-carrier for both the hops and the Wi-TIE splitting factors.

To proceed, we define a binary variable $\lambda_{k, \ell}=\{0,1\}$ to form a relay-user coupling, where $\lambda_{k, \ell}=1$ indicates the selection of $\mathcal{R}_{k}$ for $\mathcal{U}_{\ell}$ while $\lambda_{k, \ell}=0$ implies $k$-th relay is not allocated to $\ell$-th user. It is explicit that one relay is coupled with single user only, and therefore we have,

$$
\sum_{k=1}^{K} \lambda_{k, \ell}=1, \forall \ell ; \quad \sum_{\ell=1}^{L} \lambda_{k, \ell} \leq 1, \forall k .
$$

We denote $\mu_{n, \ell} \in\{0,1\}$ as the binary variable to link the first hop sub-carrier $n$ to $\mathcal{U}_{\ell}$, such that $\mu_{n, \ell}=1$ indicates that $n$ is used in the first hop to carry the relevant data for $\mathcal{U}_{\ell}$, and $\mu_{n, \ell}=0$ otherwise. In this context, the following assignment rule must be satisfied,

$$
\sum_{\ell=1}^{L} \mu_{n, \ell}=1, \quad \forall n .
$$

Correspondingly, in order to pair the sub-carriers in the two hops, let us define $\nu_{\left(n, n^{\prime}\right)} \in\{0,1\}$ as the respective indicator for sub-carrier pairing. Herein, $\nu_{\left(n, n^{\prime}\right)}=1$ implies that the sub-carrier $n$ in the first hop is paired with sub-carrier $n^{\prime}$ of the second hop, and vice-versa when $\nu_{\left(n, n^{\prime}\right)}=0$. In this regard, the following must hold,

$$
\sum_{n=1}^{N} \nu_{\left(n, n^{\prime}\right)}=1, \forall n^{\prime} ; \quad \sum_{n^{\prime}=1}^{N} \nu_{\left(n, n^{\prime}\right)}=1, \forall n .
$$

It is important to note that (C2) and (C3) automatically fixes the sub-carrier $n^{\prime}$ in the second hop for $\mathcal{U}_{\ell}$.

We impose minimum SNR constraint at the destination $\mathcal{U}_{\ell}$ corresponding to the TS and PS schemes, respectively, which are stated as follows,

$$
\begin{gathered}
\frac{1}{2}\left(1-\tau_{\ell}\right) \ln \left(1+\sum_{i=1}^{L} \sum_{k=1}^{K} \sum_{n=1}^{N} \sum_{n^{\prime}=1}^{N} \lambda_{k, \ell} \mu_{n, \ell} \nu_{\left(n, n^{\prime}\right)}\right. \\
\left.\hat{\Upsilon}_{\left(n, n^{\prime}\right), k, i}^{T S}\right) \geq \ln \left(1+\hat{\Upsilon}_{\mathcal{U}_{\ell}}\right) \\
\frac{1}{2} \ln \left(1+\sum_{i=1}^{L} \sum_{k=1}^{K} \sum_{n=1}^{N} \sum_{n^{\prime}=1}^{N} \lambda_{k, \ell} \mu_{n, \ell} \nu_{\left(n, n^{\prime}\right)} \hat{\Upsilon}_{\left(n, n^{\prime}\right), k, i}^{P S}\right) \\
\geq \ln \left(1+\hat{\Upsilon}_{\mathcal{U}_{\ell}}\right),
\end{gathered}
$$

where $\hat{\Upsilon}_{\mathcal{U}_{\ell}}$ is the minimum SNR demanded by $\mathcal{U}_{\ell}$. The factor $1 / 2$ is introduced to compensate for the two time slots of the considered relay assisted communication. Note that both the expressions in (C4) appears to be spectral efficiency constraints, but the actual decisive parameter is $\hat{\Upsilon}_{\mathcal{U}_{\ell}}$ for optimizing $\tau_{\ell}$ and $\rho_{\ell}$.

The limitations on overall power at the transmitter and the relays are respectively represented as

$$
\begin{gathered}
\sum_{n=1}^{N} p_{1, n} \leq P_{\mathcal{T}}, \\
\sum_{n^{\prime}=1}^{N} \sum_{\ell=1}^{L} \sum_{n=1}^{N} \lambda_{k, \ell} \mu_{n, \ell} \nu_{\left(n, n^{\prime}\right)} p_{2, n^{\prime}, k} \leq P_{\mathcal{R}_{k}}, k=1, \cdots, K .
\end{gathered}
$$

Considering all the intended sub-carriers at $\mathcal{U}_{\ell}$, the overall energy harvested at $\mathcal{U}_{\ell}$ is computed as follows

$$
\hat{E}_{\ell}=\sum_{k=1}^{K} \sum_{i=1}^{L} \sum_{n=1}^{N} \sum_{n^{\prime}=1}^{N} \lambda_{k, \ell} \nu_{\left(n, n^{\prime}\right)} \hat{E}_{\left(n, n^{\prime}\right), k, i} .
$$

Thus, the formulated optimization problem can subsequently be represented in its mathematical form as

$$
\begin{aligned}
(P 1): \max _{\{\boldsymbol{\lambda}, \boldsymbol{\mu}, \boldsymbol{\nu}, \mathbf{p}, \boldsymbol{\theta}\}} & \sum_{\ell=1}^{L} \hat{E}_{\ell} \\
\text { subject to : } & (C 1),(C 2),(C 3),(C 4),(C 5),(C 6), \\
& (C 7): 0 \leq \theta_{\ell} \leq 1, \ell=1, \cdots, L,
\end{aligned}
$$

where $\boldsymbol{\lambda}=\left\{\lambda_{k, \ell}\right\}, \boldsymbol{\mu}=\left\{\mu_{n, \ell}\right\}, \boldsymbol{\nu}=\left\{\nu_{\left(n, n^{\prime}\right)}\right\}, \mathbf{p}=$ $\left\{p_{1, n}, p_{2, n^{\prime}, k}\right\}$ and $\boldsymbol{\theta}=\left\{\theta_{\ell}\right\}$ denote the variables to be optimized for corresponding relay selection, carrier-destination assignment, sub-carrier pairing, sub-carrier power allocation in the two hops, and the Wi-TIE splitting factors, respectively. With an affine function (of the optimization variables) in the objective and a non-linear QoS constraint, it is difficult to obtain the optimal solution for this problem as it involves joint optimization of network resources having mixed-integer variables. However, a highly complex exhaustive search method is possible, but it is difficult to implement in practice for high parameter values as it involves a complete span over the feasible space with $(K \cdot L)^{N !}$ combinational possibilities of $\boldsymbol{\lambda}, \boldsymbol{\mu}$ and $\boldsymbol{\nu}$. In this regard, we propose an Energy Yield Escalation (EYE) method (discussed in detail in the succeeding section), with a polynomial execution time-complexity to address the problem in $(P 1)$.

\section{Proposed Solution Using the Energy Yield ESCALATION (EYE) TECHNIQUE}

In this section, we propose a novel solution summarized in the form of an Energy Yield Escalation (EYE) algorithm. For simplicity, we divide the proposed EYE algorithm into five steps: the first and second steps jointly determine the relevant binary variables $\boldsymbol{\lambda}, \boldsymbol{\mu}, \boldsymbol{\nu}$. Next, the power variable, $\mathbf{p}$, is optimized for both the hops in the third and fourth steps, respectively. Finally, the Wi-TIE splitting factors are evaluated in order to satisfy (C4), as illustrated in the fifth step. We will provide more insight on each step later in the section.

It is interesting to note that the end-to-end performance of a dual-hop AF cooperative system is associated with the harmonic mean of the SNRs in the two hops [19]. This low-complexity method of relay selection based on the the harmonic mean of the channel gains over the two hops has been proposed in literature [20], [21], and is adequately adopted due to its close to optimal yet 
IEEE International Conference on Advanced Networks and Telecommunications Systems (IEEE ANTS'18), 16-19 Dec'18, Indore, India

$$
\begin{gathered}
\tau_{\ell}=1-\frac{2 \ln \left(1+\hat{\Upsilon}_{\mathcal{U}_{\ell}}\right)}{\ln \left(1+\sum_{i=1}^{L} \sum_{k=1}^{K} \sum_{n=1}^{N} \sum_{n^{\prime}=1}^{N} \lambda_{k, \ell} \mu_{n, \ell} \nu_{\left(n, n^{\prime}\right)} \hat{\Upsilon}_{\left(n, n^{\prime}\right), k, i}^{T S}\right)} \\
\rho_{\ell}=1-\left(\frac{\left(1+\hat{\Upsilon}_{\mathcal{U}_{\ell}}\right)^{2}-1}{\sum_{i=1}^{L} \sum_{k=1}^{K} \sum_{n=1}^{N} \sum_{n^{\prime}=1}^{N} \lambda_{k, \ell} \mu_{n, \ell} \nu_{\left(n, n^{\prime}\right)} \hat{\Upsilon}_{\left(n, n^{\prime}\right), k, i}^{T S}}\right)
\end{gathered}
$$

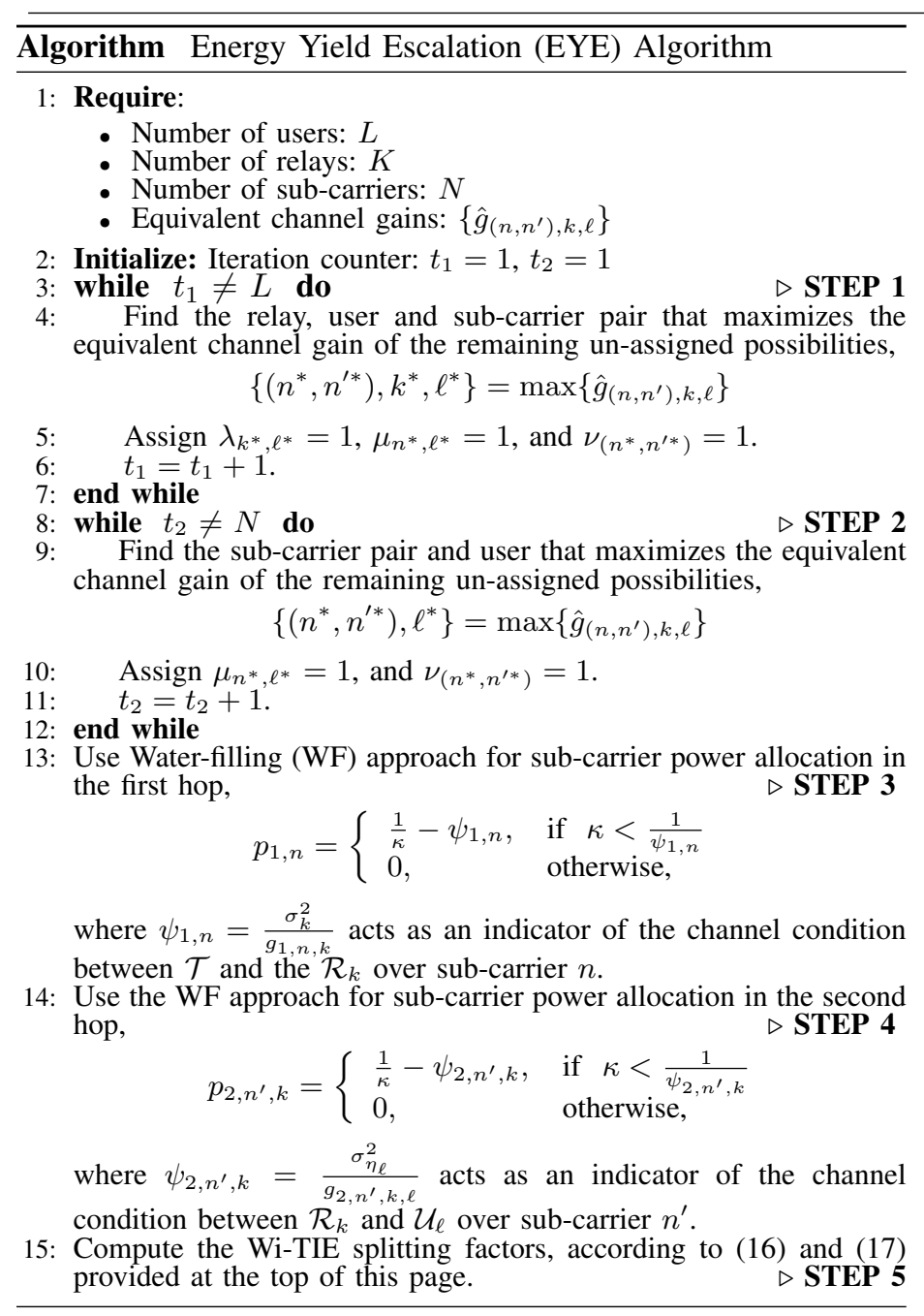

sub-optimal performance. In this regard, we define the equivalent channel gain over the $\mathcal{T} \rightarrow \mathcal{R}_{k} \rightarrow \mathcal{U}_{\ell}$ link for the $\left(n, n^{\prime}\right)$ subcarrier pair as the harmonic mean of the channel gains in the two hops, given by

$$
\hat{g}_{\left(n, n^{\prime}\right), k, \ell}=\frac{2 \cdot g_{1, n, k} \cdot g_{2, n^{\prime}, k, \ell}}{g_{1, n, k}+g_{2, n^{\prime}, k, \ell}}
$$

In this paper, we propose a sub-optimal relay and sub-carrier assignment method where we sequentially assign the relay and sub-carrier pair that maximizes the equivalent channel metric in (18). The corresponding Step 1 is summarized in the EYE Algorithm, which returns the variable $\boldsymbol{\lambda}$ but an incomplete version of the variables $\boldsymbol{\mu}$ and $\boldsymbol{\nu}$, since only the sub-carrier pairs associated with the assigned relays have been determined. To fully determine the sub-carrier pairs and assign them to the corresponding destination, we propose a similar approach where the remaining equivalent channel coefficients $\left\{\hat{g}_{\left(n, n^{\prime}\right), k, \ell}\right\}$ are sequentially maximized, taking into account the previously computed $\boldsymbol{\lambda}$ (in Step 1), as illustrated in the Step 2 of the EYE algorithm. After having obtained complete versions of the variables $\boldsymbol{\lambda}, \boldsymbol{\mu}$, and $\boldsymbol{\nu}$, we perform the power allocation to

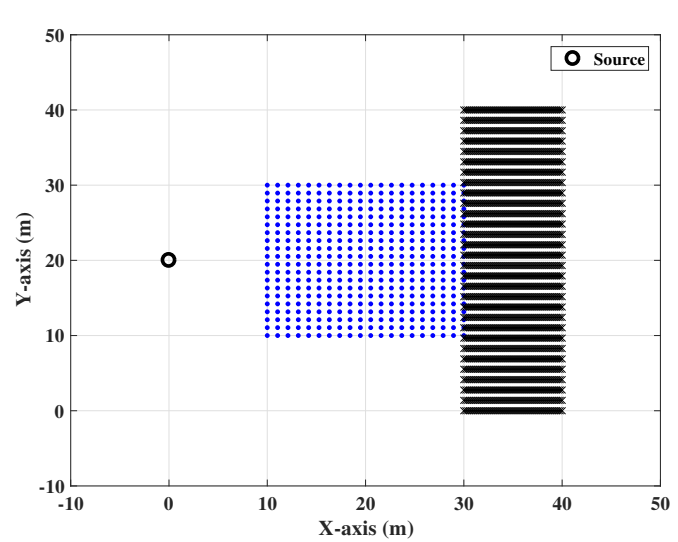

Fig. 3: Simulated scenario.

the relevant sub-carriers in the first and second hops using the conventional Water-filling (WF) approach, as mentioned in Steps 3 and 4, respectively. Finally, we compute the Wi-TIE splitting factors in Step 5 of the proposed EYE algorithm. Noticeably, the proposed mechanism offers far lower computational timecomplexity in comparison to the exhaustive search method.

\section{Computational Time-Complexity Analysis}

We present a brief analysis on the computational timecomplexities of the proposed EYE technique, the exhaustive search method, and a fixed-allocation scheme. It is noted that the proposed EYE approach with WF has a far lower execution timecomplexity of $\mathcal{O}\left(K \cdot L^{2} \cdot N^{3}\right)$, in comparison to an optimal exhaustive search approach with $(K \cdot L)^{N !}$ possible combinations of $\boldsymbol{\lambda}, \boldsymbol{\mu}$, and $\boldsymbol{\nu}$. The exhaustive search method becomes inconvenient to realize at higher values of $K, L$, and $N$, due to extremely high execution time-complexity. However, the computational timecomplexity of the fixed-allocation method, where the relays and sub-carriers are assigned at random, followed by $\mathrm{WF}$, is very fast and of $\mathcal{O}\left(N^{2}\right)$. However, using this kind of hit-and-trial technique has the probability of getting the optimal choice as $1 /(K \cdot L)^{N !}$, which becomes immensely small at higher parameter values. Therefore, the fixed-allocation method is considered to provide a sub-optimal solution unless the hit-and-trial method coincides exactly with optimal selection, which is highly unlikely in practice.

\section{Simulation Results}

In this section, we demonstrate the effectiveness of the proposed EYE technique using simulations. Various parametric alterations help in testing the efficacy of the proposed method over different emulations. We assume that the transmit powers at $\mathcal{T}$ and at each relay are constrained to the same limit i.e., $P_{\mathcal{T}}=P_{\mathcal{R}_{1}}=\ldots=P_{\mathcal{R}_{K}}=P$, same SNR demand at each user i.e., $\hat{\Upsilon}_{\mathcal{U}_{1}}=\hat{\Upsilon}_{\mathcal{U}_{2}}=\ldots=\hat{\Upsilon}_{\mathcal{U}_{L}}$, and $\zeta=0.8$ throughout this paper. Each emulation point in all the figures correspond to the sum of harvested energy at each user obtained after averaging over 500 Monte-Carlo random channel experimental realizations. 


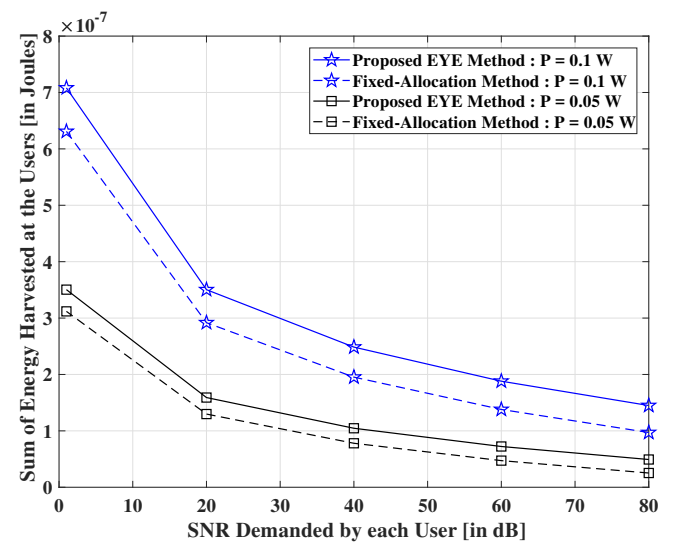

(a)

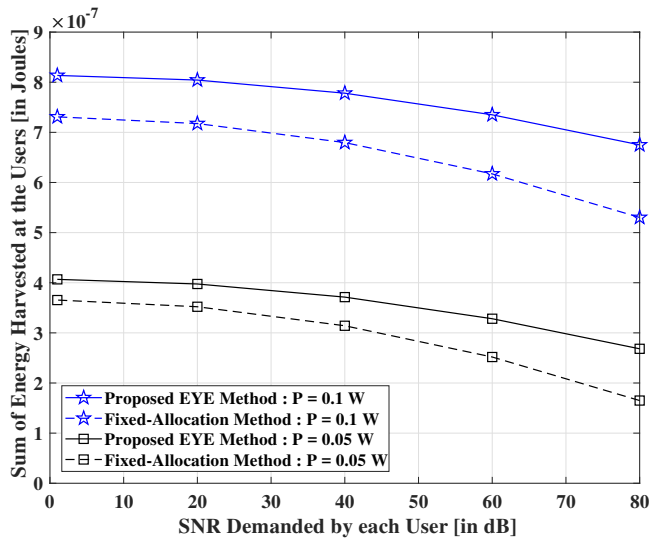

(b)

Fig. 4: Sum of the energy harvested at each user versus different SNR demands for various values of transmit powers with $K=3$, $L=2$, and $N=32$ to investigate the efficiency of (a) TS scheme, and (b) PS scheme.

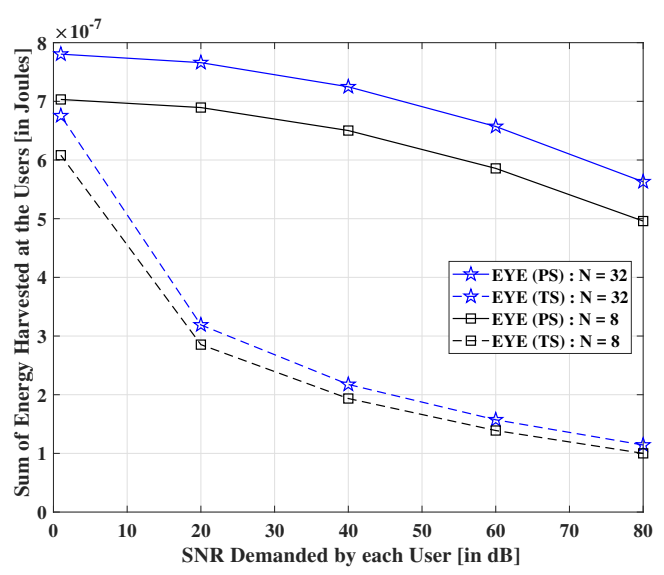

Fig. 5: Frequency diversity gain with $K=6, L=4$.

Considering the placement of nodes as depicted in Fig. 3, we assume that a transmitter is placed at $(0,20) \mathrm{m}$ in the system with $K$ relays spatially distributed within a $20 \times 20 \mathrm{~m}^{2}$ area i.e., inside the square coordinates $(10,10) \mathrm{m}$ and $(30,30) \mathrm{m}$, and the endusers are randomly located within a $400 \mathrm{~m}^{2}$ rectangular region between the coordinates $(30,0) \mathrm{m}$ and $(40,40) \mathrm{m}$. In order to simulate a wireless broadband network with $802.11 \mathrm{~b} / \mathrm{g}$ or similar type of access point, we employ the ITU Radiocommunication Sector (ITU-R) P.1238 channel model. In this frequency-selective channel model, the central frequency is assumed at $1.9 \mathrm{GHz}$ with the mean of 5-multipath arrivals obtained via Poisson process and the root mean square (rms) delay of $36.3078 \mathrm{~ns}$ for the specified room dimensions. The signal fading in both the hops follow the Ricean distribution with the K-factor of 3.5. Assuming an overall bandwidth of $20 \mathrm{MHz}$, each OFDMA sub-carrier is assumed to experience a flat-fading with negligible impact from shadowing, which is therefore discarded in the system set-up. We assume the noise power at the relay and user nodes to be the same i.e., -90 $\mathrm{dBm}[22]$.

We consider a fixed-allocation method as the benchmark where the relays and sub-carriers are assigned at random followed by the sub-carrier power allocation and computation of Wi-TIE splitting factors using Steps 3 to 5 of the proposed EYE algorithm. Since the exhaustive search method is highly complex and difficult to realize in practice for high parameter values and due to the lack of prior work in this particular problem, we choose the fixedallocation approach for comparison purposes. Fig. 4(a) and Fig.

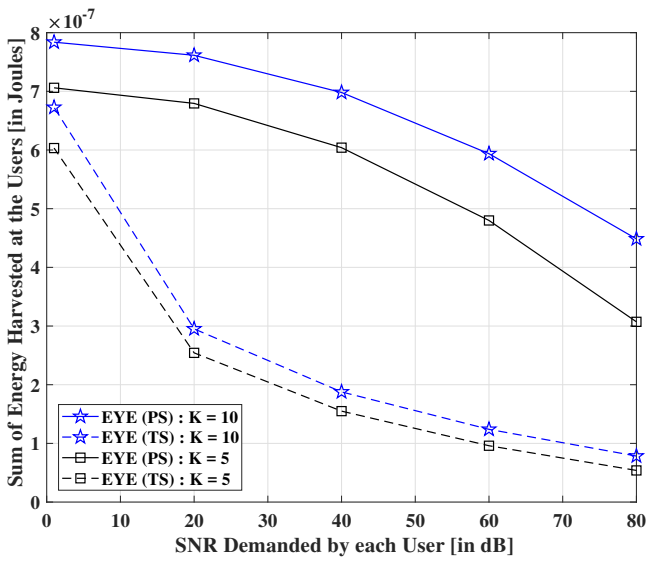

Fig. 6: Relays' diversity gain with $L=5, N=32$.

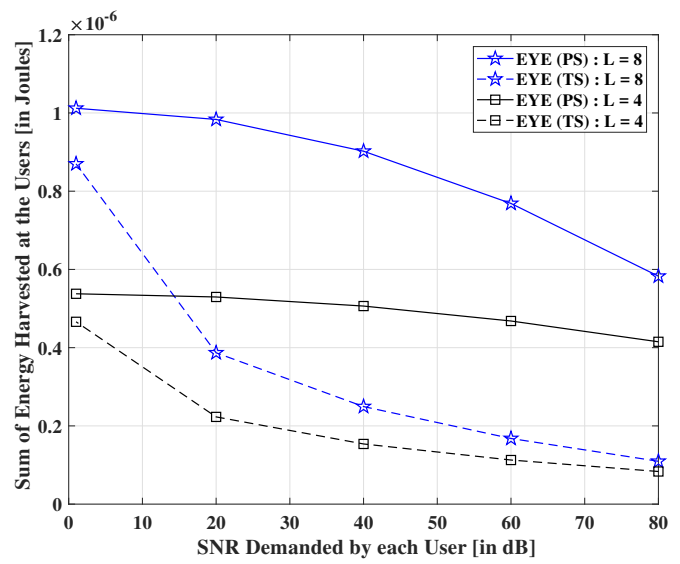

Fig. 7: Users' diversity gain with $K=12, N=32$.

4(b) compares the sum of energy harvested at the users versus the demanded SNR values at each user with TS and PS architectures, respectively, using the proposed EYE method and the fixedallocation strategy in a network composed of $K=3$ relays and $L=2$ users, with $N=32$ OFDMA sub-carriers for different transmit power values. Different location of relays and the users are selected to realize various channel coefficients. We observe that the proposed EYE method performs considerably better than the fixed-allocation technique with PS receiver architecture outperforming the TS scheme in both the approaches. It is also noted that the performance of the system improves significantly with increasing transmit power values, however, the sum of energy 
harvested by the user decreases with increasing SNR demand at each user.

Next, we demonstrate in Fig. 5 the impact of frequency diversity on the considered system with the help of the proposed EYE technique. We set $K=6, L=4, N=32$ and $P=0.075 \mathrm{~W}$. It is observed that the system performance increases appreciably with higher number of OFDMA sub-carriers. In addition, the sum of energy harvested by the users depreciates non-linearly with the high SNR demands at each user for both TS and PS Wi-TIE architectures. The PS-based Wi-TIE scheme provides an outstanding performance in comparison to its counterpart TS method for the two test cases of $N=8$ and $N=32$. Moreover, it is noteworthy that the efficiency of the PS-based Wi-TIE receiver improves tremendously with increasing number of sub-carriers.

In Fig. 6, we investigate the gain provided by the diversity in the available relays with the network comprising of $L=5$, $N=32$, and $P=0.05 \mathrm{~W}$. We notice an improvement in the system performance when the number of available relays is high. Instinctively, more number of relays provide adequate diversity to improve the overall system performance. The sum of energy harvested at the users is found to be decreasing with increasing SNR demand at each user while the PS continue to provide better results in comparison to the TS Wi-TIE architecture.

Fig. 7 illustrates the efficacy of the proposed EYE algorithm with varying number of users for $K=12, N=32$, and $P=0.075 \mathrm{~W}$. Intuitively, with increasing number of users, more relays will be invoked and an improvement is expected in the system as a consequence. Assuming sufficient network resources are available, we show the improvement in the system performance for increasing number of users. However, as mentioned previously, the sum of energy harvested at the users depletes with growing SNR demands at each user. On the other hand, as mentioned before, it is noteworthy that PS outperforms TS in all the considered system setups.

\section{CONCLUSION}

In this paper, we investigated resource allocation and relay selection in a half-duplex multi-carrier multi-user wireless cooperative system, where the relays employ the AF protocol and the end-users are equipped with Wi-TIE capabilities. We maximized the total energy harvested at the users under constraints on minimum SNR at each of the end-users and on the transmit powers at the transmitter and relays. In addition, this problem was studied for both TS and PS Wi-TIE schemes. In this context, we proposed an Energy Yield Escalation (EYE) algorithm with polynomial computation time-complexity and sub-optimal but good performance, to address the aforementioned problem. We illustrated the efficiency of the proposed technique via numerical results. This work can be extended to many interesting directions like considering this framework with Non-Orthogonal Multiple Access (NOMA), and multi-antenna based systems.

\section{ACKNOWLEDGMENT}

This work was partially supported by the Luxembourg National Research Fund (FNR) in the framework of the FNR-FNRS bilateral project "InWIP-NET : Integrated Wireless Information and Power Networks".

\section{REFERENCES}

[1] T. D. P. Perera, D. N. K. Jayakody, S. K. Sharma, S. Chatzinotas, and J. Li, "Simultaneous Wireless Information and Power Transfer (SWIPT): Recent Advances and Future Challenges," IEEE Communications Surveys \& Tutorials, 2017.

[2] X. Zhou, R. Zhang, and C. K. Ho, "Wireless Information and Power Transfer: Architecture Design and Rate-Energy Tradeoff," IEEE Trans. Commun., vol. 61, no. 11, pp. 4754-4767, Nov. 2013.

[3] S. Gautam and P. Ubaidulla, "Simultaneous transmission of information and RF energy in multicarrier systems," in 2016 23rd International Conference on Telecommunications (ICT). IEEE, 2016, pp. 1-5.

[4] B. Nikfar and A. J. Han Vinck, "Relay selection in cooperative power line communication: A multi-armed bandit approach," Journal of Communications and Networks, vol. 19, no. 1, pp. 1-9, February 2017.

[5] T. Cover and A. E. Gamal, "Capacity theorems for the relay channel," IEEE Transactions on Information Theory, vol. 25, no. 5, pp. 572-584, September 1979.

[6] J. N. Laneman, D. N. C. Tse, and G. W. Wornell, "Cooperative diversity in wireless networks: Efficient protocols and outage behavior," IEEE Transactions on Information Theory, vol. 50, no. 12, pp. 3062-3080, December 2004.

[7] Y. Chen, R. Shi, W. Feng, and N. Ge, "AF Relaying With Energy Harvesting Source and Relay," IEEE Transactions on Vehicular Technology, vol. 66, no. 1, pp. 874-879, Jan 2017.

[8] W. Lu, Y. A. Zhang, M. Wang, X. Liu, and J. Hua, "Cooperative Spectrum Sharing in OFDM Two-Way Relay Systems With Bidirectional Transmissions," IEEE Communications Letters, vol. 21, no. 6, pp. 13491352, June 2017.

[9] A. El Shafie, K. Tourki, and N. Al-Dhahir, "An Artificial-Noise-Aided Hybrid TS/PS Scheme for OFDM-Based SWIPT Systems," IEEE Communications Letters, vol. 21, no. 3, pp. 632-635, March 2017.

[10] L. Liu, R. Zhang, and K. C. Chua, "Wireless Information Transfer with Opportunistic Energy Harvesting," IEEE Transactions on Wireless Communications, vol. 12, no. 1, pp. 288-300, January 2013.

[11] Y. Lu, K. Xiong, Y. Zhang, P. Fan, and Z. Zhong, "Energy-Efficient Resource Allocation in OFDM Relay Networks under Proportional Rate Constraints," in 2016 IEEE Global Communications Conference (GLOBECOM), Dec 2016, pp. 1-6.

[12] S. Khakurel, L. Musavian, H. V. Vu, and T. Le-Ngoc, "QoS-Aware UtilityBased Resource Allocation in Mixed-Traffic Multi-User OFDM Systems," IEEE Access, pp. 1-1, 2018.

[13] K. Xiong, P. Fan, C. Zhang, and K. Letaief, "Wireless Information and Energy Transfer for Two-Hop Non-Regenerative MIMO-OFDM Relay Networks," IEEE J. Sel. Area Commun., vol. 33, no. 8, pp. 1595-1611, Aug. 2015.

[14] S. Gautam, E. Lagunas, S. K. Sharma, S. Chatzinotas, and B. Ottersten, "Relay Selection Strategies for SWIPT-Enabled Cooperative Wireless Systems," IEEE Int. Symp. Personal, Indoor and Mobile Radio Communications (PIMRC), Montreal, Canada, Oct. 2017.

[15] S. Gautam and P. Ubaidulla, "Relay Selection and Transceiver Design for Joint Wireless Information and Energy Transfer in Cooperative Networks," in 85th Vehicular Technology Conference (VTC), 2017-Spring, June 2017.

[16] L. Liu, R. Zhang, and K. C. Chua, "Wireless Information and Power Transfer: A Dynamic Power Splitting Approach," IEEE Trans. Commun., vol. 61, no. 9, pp. 3990-4001, Sep. 2013.

[17] Y. Li, W. Wang, J. Kong, and M. Peng, "Subcarrier Pairing for Amplify-andForward and Decode-and-Forward OFDM Relay Links," IEEE Commun. Lett., vol. 13, no. 4, pp. 209-211, Apr. 2009.

[18] W. Dang, M. Tao, H. Mu, and J. Huang, "Subcarrier-Pair Based Resource Allocation for Cooperative Multi-Relay OFDM Systems," IEEE Trans. Wireless Commun., vol. 9, no. 5, pp. 1640-1649, May 2010.

[19] M. O. Hasna and M. S. Alouini, "End-to-End Performance of Transmission Systems with Relays Over Rayleigh-Fading Channels," IEEE Trans. Wireless Commun., vol. 2, no. 6, pp. 1126-1131, Nov. 2003.

[20] A. Bletsas, A. Khisti, D. P. Reed, and A. Lippman, "A Simple Cooperative Diversity Method Based on Network Path Selection," IEEE J. Sel. Areas Commun., vol. 24, no. 3, pp. 659-672, Mar. 2006.

[21] S. Biyanwilage, U. Gunawardana, and R. Liyanapathirana, "Selective SubCarrier Relaying and Power Allocation for Multi-Relay-Assisted Cooperative OFDM Systems with Outdated CSI," Int. Conf. on Telecommunications (ICT), Ayia Napa, Cyprus, pp. 528-533, May. 2011.

[22] Jim Geier, "How to: Define Minimum SNR Values for Signal Coverage," http://www.wireless-nets.com/resources/tutorials/define_SNR_values. html, Wireless-Nets, 2013, [Online]. 